\title{
Sensorless control of Switched Reluctance Motor for EV Application using a Sliding Mode Observer with Unknown Inputs
}

\author{
Yakoub SAADI \\ ESTACA Engineering School \\ Laval, France \\ yakoub.saadi@estaca.fr \\ Mousaa BOUKHNIFER \\ ESTACA Engineering School \\ Paris, France \\ moussa.boukhnifer@estaca.fr
}

\author{
Rabia SEHAB \\ ESTACA Engineering School \\ Laval, France \\ rabia.sehab@estaca.fr
}

\author{
Ahmed CHAIBET \\ ESTACA Engineering School \\ Paris, France \\ ahmed.chaibet@estaca.fr \\ Demba DIALLO \\ Group of Electrical Engineering - Paris (GeePs) \\ Paris, France \\ demba.diallo@geeps.centralesupelec.fr
}

\begin{abstract}
This paper deals with the sensorless position control of 8/6 Switched Reluctance Machine used in the drivetrain of an Electric Vehicle. The developed observer could be an excellent alternative to replace the analog position sensor, in case of high maintenance cost, limit lifecycle and safety-driving with a sensor default. The software position sensor based on Sliding Mode Observer consists of estimating the rotor position, the velocity and the torque of the SRM drive with a known and an unknown load torque. The main advantage of the developed observer is to estimate online the variables over all the velocity operation range using only the current and voltage measurements of each phase. However, the proposed observer is implemented in a simulator, where results confirm the reliability and the accuracy of the developed observer comparing to the real rotor position, velocity and machine torque.
\end{abstract}

Index Terms-Electric Vehicle, Switched Reluctance Machine, position sensorless control, Sliding Mode Observer, rotor position estimation, velocity estimation, machine torque estimation.

\section{INTRODUCTION}

$\mathcal{M}$ ODERN vehicles have become more and more electrified, in particular, Electric Vehicles (EVs) which gaining increased attention due to environmental and energy concerns. In the vehicle drivetrain, the main component is the electric machine. In such an application, high power density, high torque density, wide speed range, and efficiency are of primary importance [1]. To meet these demands, Rare Earth Magnetic Material (REMM) has been widely used in different types of electric machines dedicated to the EVs applications like the sintered Neodymium Iron Boron and Samarium Cobalt [2]. However, the cost of REMM-based machines has increased over several years. Moreover, due to the limited resources, the use of REMMbased machines in EVs applications is now being challenged. In fact, many researchers are working on other machines that can be competitive regarding size, efficiency and torque density.
Considering the requirements above, Switched Reluctance Machines (SRMs) represent an alternative. It does not only features a salient pole stator with concentrated coils, which provides earlier winding and shorter end turn than other types of machines but also features a salient pole rotor, which has neither conductors nor magnets. Simplicity in its construction makes the SRM inexpensive. Its high reliability and performance at high speeds, its fault-tolerant operation capability and the simplicity of the power converter make it an attractive candidate for electric vehicles propulsion. However, the SRM exhibits high torque ripples and acoustic noises. Nevertheless, these drawbacks can be significantly reduced with an optimal mechanical design [3] and a good control strategy [4] [5].

For EVs, sensorless control of electric machines is a particularly relevant topic. Its purpose is to eliminate the mechanical position sensor and extract the rotor position information indirectly from electrical measurements and processing algorithms. It will contribute to reducing the maintenance cost, the size package of the drivetrain and ensure safe driving in case of sensor default. It is also independent of sensor signal distortion and electromagnetic interference, therefore, increasing the reliability. The sensorless control should use only available data measured from machine terminals and be effective over the entire speed and torque range with high resolution and accuracy [6].

In the literature, various methods of sensorless control for SRMs have been investigated in the last twenty years, with their advantages and disadvantages. Almost most of these techniques rely on the same central idea, that is, to recover the mechanical position from the inductance, the flux-linkage, the phase current or the back electromotive force(EMF). The initial results of these methods, reported by 
Lumsdaine and Lang [7], were highly encouraging.. However, because a large storage memory is required, numerical methods are widely reported to calculate the instantaneous inductance or the instantaneous flux of the machine [12], but lots of computational efforts are still needed during operation. Recently, several studies have been done using artificial intelligence techniques such as the Artificial Neural Networks(NNs) [8] and Fuzzy Neural Networks (FNNs) [9]. Nowadays, with the expeditious progress in digital signal processor (DSP) and the microprocessor technology, observer-based sensorless algorithms are becoming attractive.

Sliding Mode Observer (SMO), with its benefits of high stability, computational simplicity and robustness properties, provides a powerful approach to implement an observer-based sensorless algorithm for the EVs powertrain by the SRM. Nonetheless, sliding mode observers often fail at very low-speed because no driving signals are available for the observer to estimate the rotor position [10].

In this context, the authors propose a SMO for sensorless control of SRMs drives over the entire speed range with a new sliding surface is adopted. The main contribution is the reduction of the estimation errors at very low-speed (near zero) and at a standstill. At first, a SMO will be developed and tested when the load torque is supposed to be known. Then the unknown load torque case will be treated.

The paper is organized as follows: The model of the SRM is given in section II. A short description of the SMO basedobserver for the electric vehicles scheme is given in section III. Section III-B is devoted to present the design of SMO with a known load torque. The design of SMO with unknown load torque is presented in section III-C. Conclusion and perspectives are being drawn in section IV.

\section{MODELLING OF THE SRM}

The SRMs have a simple construction, but the solution of its mathematical models is relatively complicated due to its dominant nonlinear behavior. The flux-linkage is a function of two variables, the current and the rotor position. The SRM used in this study has 4 phases, it is a 8/6-type machine. The flux-linkages of one phase is presented in Fig.(1) and the parameters of the SRM are given in the appendix. The mathematical model of the equivalent circuit is given by [5]:

$$
V_{j}=R_{j} I_{j}+\frac{\partial \psi_{j}(\theta, I)}{\partial t}
$$

With $j=1,2,3,4$.

The electromagnetic dynamic model of the SRM and load can be expressed as follows:

$$
\left\{\begin{array}{c}
\frac{\mathrm{d} \Omega}{\mathrm{dt}}=\frac{1}{J}\left(T_{e}(\theta, I)-f_{r} \Omega-T_{L}\right) \\
J=J_{\text {load }}+J_{S R M}
\end{array}\right.
$$

The instantaneous torque developed by the phase $j$ is [5]

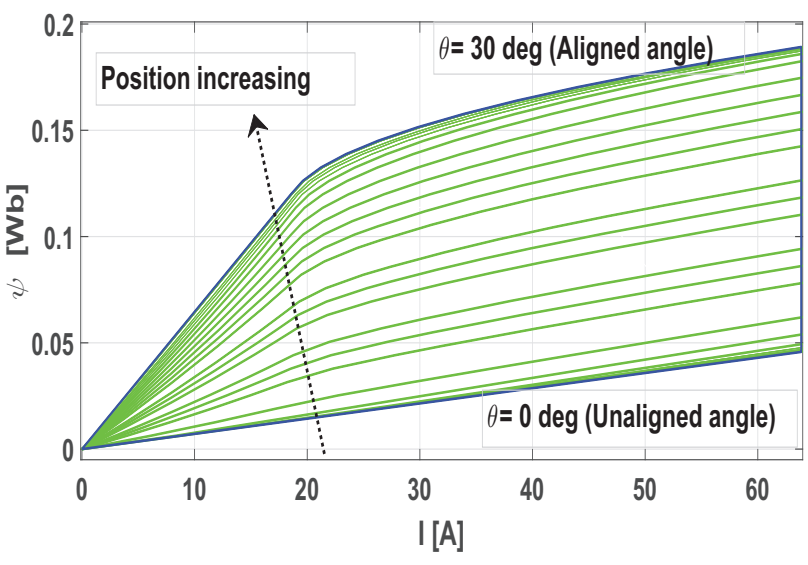

Figure 1: Flux-linkage of the $S R M$.

Nomenclature:

$\begin{array}{llll}\theta & \text { Rotor position } & I_{j} & \text { Current in the } j^{t h} \text { phase } \\ \Omega & \text { Angular velocity of rotor } & \psi_{j} & \text { Flux-linkages in } j^{t h} \text { phase } \\ T_{e} & \text { Total electromagnetic torque } & R_{j} & \text { Resistance of the } j^{t h} \text { phase } \\ f_{r} & \text { Friction coefficient } & L & \text { Instantaneous inductance } \\ J & \text { Total inertia (rotor and load) } & V_{j} & \text { Voltages of the } j^{t h} \text { phase } \\ J_{l o a d} & \text { Vehicle inertia } & J_{S R M} & \text { Machine inertia } \\ T_{L} & \text { Load torque } & & \end{array}$

$$
T_{\text {phase }_{j}}=\frac{1}{2} \frac{\mathrm{dL}(\theta)}{\mathrm{d} \theta} I_{j}^{2}
$$

The equation (3) applies only in the case of magnetic linearity. In this research, the $T_{\text {phase }_{j}}$ is calculated by using the torquelinkage which is a nonlinear function of the stator phase current and rotor position.

The instantaneous torque developed by the machine is the sum of the instantaneous torques developed by each phase:

$$
T_{e}=\sum_{j=1}^{j=4} T_{\text {phase }_{j}}
$$

\section{SLIDING MODE OBSERVER DESIGN FOR EVS APPLICATION}

\section{A. Introduction}

The primary objective of this section is to replace the position sensor by a SMO to carry out the sensorless control of the EV propelled by a SRM. The observer is in charge of estimating the position of the SRM drive over the entire speed range using only the current and voltage measurements of each phase. At first, a SMO will be developed and tested when the load torque is supposed to be known. Then the unknown load torque case will be treated.

The Fig.(2) shows the cascade control scheme approach adopted in this study and the implementation of the SMO. The outer control loop provides the total reference torque through PI speed controller, which is regulated indirectly by the inner control loops through the four PI current controllers.

In this research, the shaft (load) inertia moment is considered negligible compared to the SRM inertia moment $\left(J \approx J_{S R M}\right)$. 


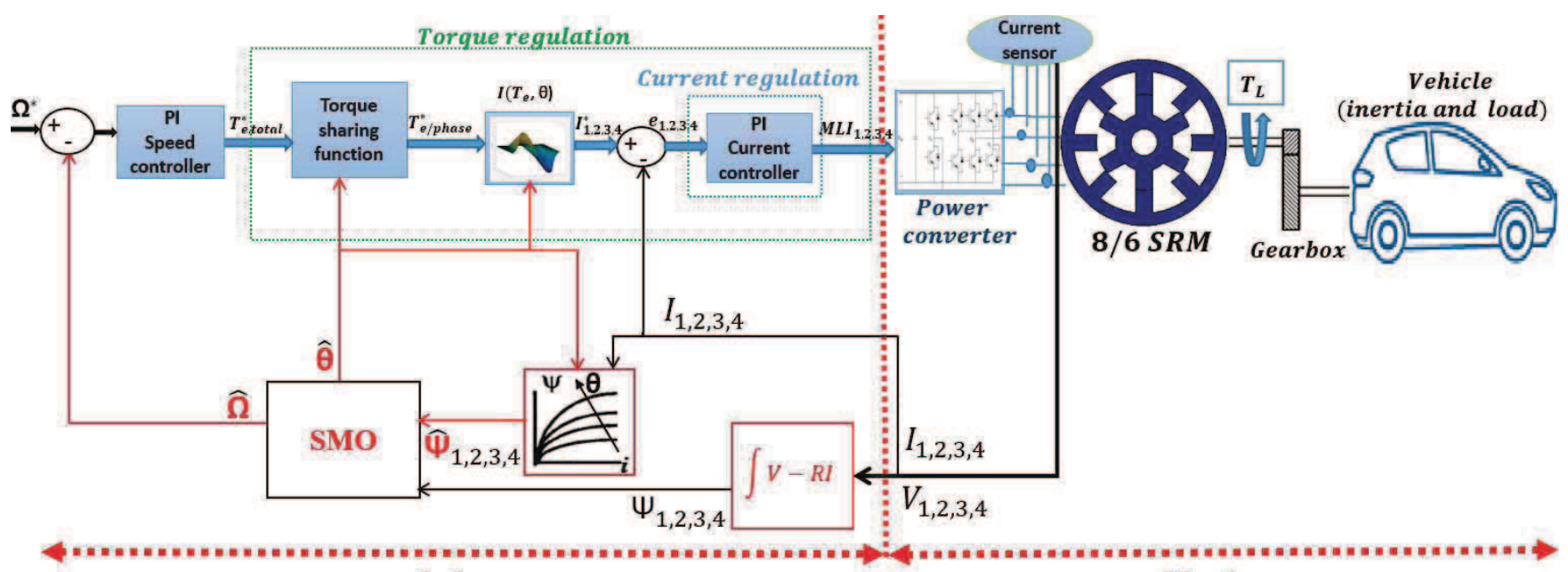

Software

Hardware

Figure 2: Implementation of the Sliding Mode Observer in the vehicle drivetrain.

\section{B. Sliding Mode Observer with known load torque}

According to the dynamic model (2), a SMO for rotor position, speed and torque estimation can be defined as follows:

$$
\left\{\begin{array}{c}
\frac{\mathrm{d} \hat{\theta}}{\mathrm{dt}}=\hat{\Omega}+K_{\theta} \operatorname{sign}(S) \\
\hat{T}_{e}=T_{e}(\hat{\theta}, I) \\
\frac{\mathrm{d} \hat{\Omega}}{\mathrm{dt}}=\frac{1}{J}\left(\hat{T}_{e}-f_{r} \hat{\Omega}-T_{L}\right)+K_{\Omega} \operatorname{sign}(S)
\end{array}\right.
$$

Where $\hat{\theta}, \hat{\Omega}$ and $\hat{T}_{e}$ are the estimations of $\theta, \Omega$ and $T_{e}$ respectively. $K_{\theta}$ and $K_{\Omega}$ are the SMO gains. $S$ is the SMO switching surface which compares measured electrical variables with their corresponding estimated values it will be discussed in the next subsection(III-B1). $\hat{T}_{e}$ is calculated from the injection of the current phase and the estimated position in torque-linkage of the SRM.

It is clear that if $\hat{\theta} \longrightarrow \theta$, so $\hat{T}_{e} \longrightarrow T$, then $\hat{\Omega} \longrightarrow \Omega$.

The estimation error of rotor position $e_{\theta}$ and the estimation error of speed $e_{\Omega}$ are defined as:

$$
\left\{\begin{array}{r}
e_{\theta}(t)=\theta(t)-\hat{\theta}(t) \\
e_{\Omega}(t)=\Omega(t)-\hat{\Omega}(t)
\end{array}\right.
$$

The error dynamics can be obtained by differentiating (6) as follows:

$$
\left\{\begin{array}{r}
\dot{e}_{\theta}=e_{\Omega}-K_{\theta} \operatorname{sign}(S) \\
\dot{e}_{\Omega}=\frac{1}{J}\left(T_{e}-\hat{T}_{e}\right)-\frac{f_{r}}{J} e_{\Omega}-K_{\Omega} \operatorname{sign}(S)
\end{array}\right.
$$

In order to find the observer gains that ensure the stability of the proposed SMO, a Lyapunov theorem for asymptotic stability is introduced [11]. For $K_{\theta}$, we define the following positive definite Lyapunov function:

$$
V_{\theta}=\frac{1}{2} e_{\theta}^{2}
$$

The derivative of the Lyapunov function is:

$$
\dot{V}_{\theta}=e_{\theta} \dot{e}_{\theta}=e_{\theta} e_{\Omega}-K_{\theta} e_{\theta} \operatorname{sing}(S)
$$

To keep the derivative of the Lyapunov function negative definite, two conditions have to be satisfied:

- $S$ must have the same sign as $e_{\theta}$

- $K_{\theta}>\left|e_{\Omega}\right|$

As there's no rotor position sensor, $\theta$ and $\Omega$ cannot be measured and $e_{\Omega}$ is uncertain. However, through the knowledge of the technical characteristics of the machine, the maximum value of speed error $\left(\left|e_{\Omega}\right|_{\max }\right)$ could be assumed. The velocity estimation error must satisfy $e_{\Omega}<\left|e_{\Omega}\right|_{\max }$. Then, the position observer gain $K_{\theta}$ can be designed as:

$$
K_{\theta}>\left|e_{\Omega}\right|_{\max }
$$

Using the same Lyapunov function as defined previously in (8), we can write for the speed dynamic error:

$$
V_{\Omega}=\frac{1}{2} e_{\Omega}^{2}
$$

Moreover, the derivative of $V_{\Omega}$ is:

$$
\dot{V}_{\Omega}=\frac{1}{J}\left(T_{e}-\hat{T}_{e}\right) e_{\Omega}-\frac{f_{r}}{J} e_{\Omega}^{2}-e_{\Omega} K_{\Omega} \operatorname{sign}(s)
$$

If $K_{\Omega}$ is chosen high enough, the first term in (12) can be neglected:

$$
\dot{V}_{\Omega}=-\frac{f_{r}}{J} e_{\Omega}^{2}-e_{\Omega} K_{\Omega} \operatorname{sign}(S)
$$

Replacing $e_{\Omega}$ from the first equation of (7) in (13):

$$
\dot{V}_{\Omega}=-\frac{f_{r}}{J} e_{\Omega}{ }^{2}-\dot{e}_{\theta} K_{\theta} K_{\Omega} \operatorname{sing}(S)-K_{\theta} K_{\Omega} \operatorname{sing}(S)^{2}
$$

Since the gain $K_{\theta}$ is chosen to satisfy (10), the sliding surface $e_{\theta}=0$ will be reached in finite time, then, $\dot{e}_{\theta}=0$.

To ensure that $\dot{V}_{\Omega}$ is negative definite, $K_{\Omega}$ must verify:

$$
K_{\Omega}>0
$$




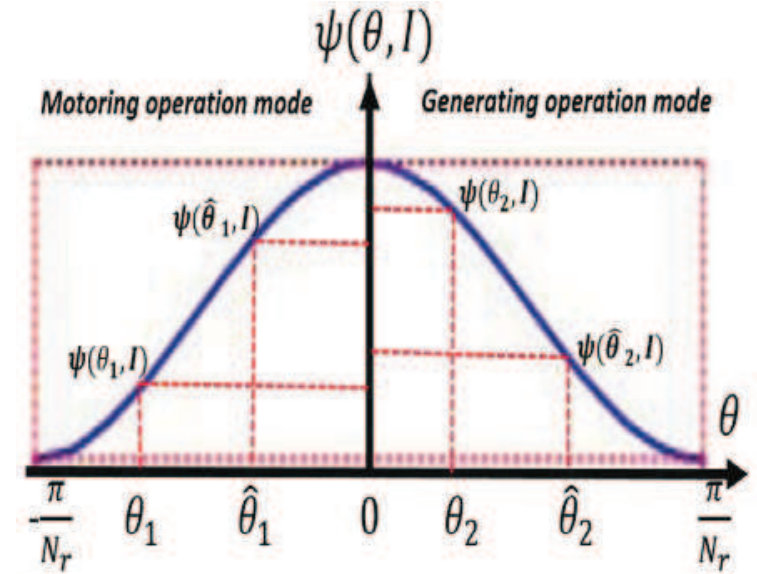

Figure 3: Phase flux profile.

1) Sliding surface design and analysis: From the above analysis, we get to the conclusion that the sliding surface $(S)$ should have the same sign as $e_{\theta}$. To meet this requirement, the following surface function is proposed:

$$
S=\sum_{i=1}^{N} \sin \left[N_{r}\left(\hat{\theta}-(i-1) \frac{2 \pi}{N N_{r}}\right)\right]\left(\hat{\psi}_{i}-\psi_{i}\right)
$$

Where, $N$ and $N_{r}$ are the number of phases and number of rotor poles respectively.

To prove that the sliding surface $S$ has the same sign as $e_{\theta}$ we analyse the sign of $S$ for the two operating modes of the machine; motoring and generating. Fig.(3) shows the flux profile in one phase of the SRM.

It can be observed that when the rotor position $\theta$ lies between $\left[-\frac{\pi}{N_{r}}, 0\right]$, the flux increases and the SRM operates in motor mode, and we have :

$$
\sin \left[N_{r}\left(\hat{\theta}-(i-1) \frac{2 \pi}{N N_{r}}\right)\right]<0
$$

Two cases have to be discussed:

- if $\hat{\theta}>\theta$, then $e_{\theta}=\theta-\hat{\theta}<0$, in this case, $\hat{\psi}>\psi$ and $S=\sum \sin ().(\hat{\psi}-\psi)<0$

- elseif $\hat{\theta}<\theta$, then $e_{\theta}=\theta-\hat{\theta}>0$, in this case, $\hat{\psi}<\psi$ and $S=\sum \sin ().(\hat{\psi}-\psi)>0$

So in motor mode, $e_{\theta}$ and $S$ have the same sign.

When $\theta$ lies between $\left[0, \frac{\pi}{N_{r}}\right]$, the flux decreases and the machine operates in generator mode. We can notice that :

$$
\sin \left[N_{r}\left(\hat{\theta}-(i-1) \frac{2 \pi}{N N_{r}}\right)\right]>0
$$

With the same analysis as above:

- if $\hat{\theta}>\theta$, then $e_{\theta}=\theta-\hat{\theta}<0$, in this case, $\hat{\psi}<\psi$ and $S=\sum \sin ().(\hat{\psi}-\psi)<0$

- else $\hat{\theta}<\theta$, then $e_{\theta}=\theta-\hat{\theta}>0$, in this case, $\hat{\psi}>\psi$ and $S=\sum \sin ().(\hat{\psi}-\psi)>0$

In summary, the sliding $\operatorname{surface}(S)$ defined in (16) has always the same sign as $e_{\theta}$.

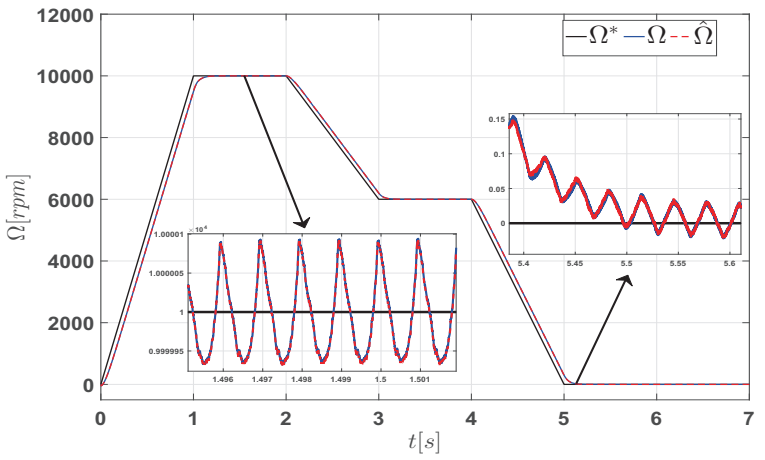

Figure 4: The rotor speed estimation.

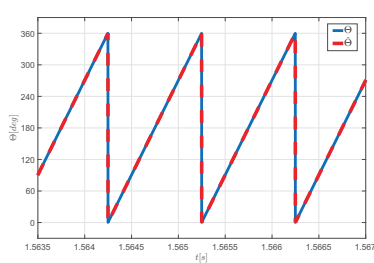

(a) At maximum speed.

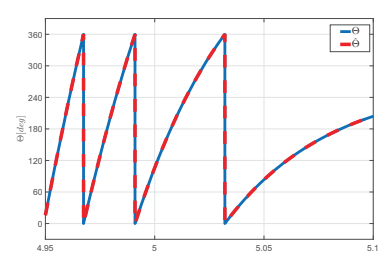

(b) At very low-speed.
Figure 5: The rotor position estimation.

2) Simulation results: In this section, simulations are performed to evaluate the proposed SMO. The objectives are to estimate the rotor position, the speed, the machine torque and to minimize the estimation errors. A speed profile which covers the entire speed range from zero to maximum speed (10000rpm) is proposed as a scenario. In the first case, a constant load torque $T_{L}=16 \mathrm{Nm}, 80 \%$ of the nominal torque is applied for the whole simulation. To minimize chattering, a boundary layer along sliding manifold is introduced by replacing the function $\operatorname{sign}(S)$ in $(5)$ by the $\operatorname{sat}(S)$ function, where:

$$
\operatorname{sat}(S)=\left\{\begin{array}{c}
-1, S<\phi \\
\frac{S}{\phi},-\phi \leq S \leq \phi \\
1, S>\phi \\
0<\phi<1
\end{array}\right.
$$

The SMO parameters used in this simulation are: $K_{\theta}=750$, $K_{\Omega}=250$ and $\phi=0.5$.

Fig.(4) displays the $\operatorname{refernce}\left(\Omega^{*}\right)$, estimated $(\hat{\Omega})$ and actual $\operatorname{speed}(\Omega)$. Fig.(6) shows the speed estimation error. As it can be seen, the estimated speed converges on the whole profile including at a standstill. Fig.(7) shows the convergence of the sliding surface to zero. Fig.(5) shows the efficacy of the SMO to estimating the rotor position at maximum and even at very low speed and standstill.

The electromagnetic torque of the machine and its estimated are plotted in Fig.(8), from these results we can conclude that when the load torque is known, the SMO exhibits good performances in the estimation of mechanical variables and the electromagnetic torque. 


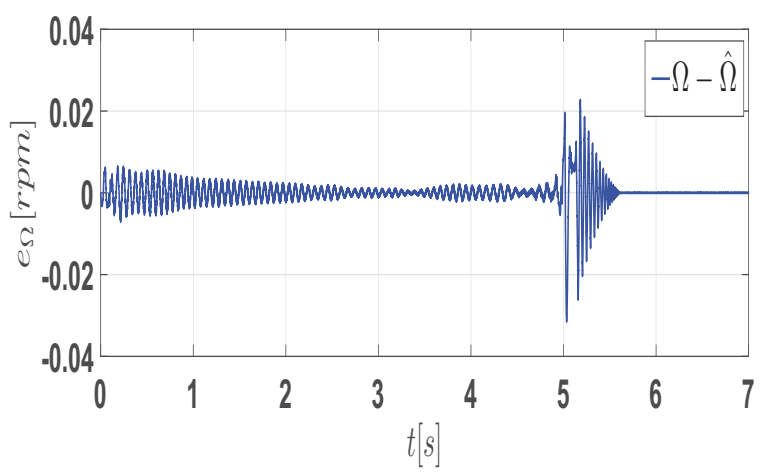

Figure 6: The estimation error of the speed $\left(e_{\Omega}\right)$.

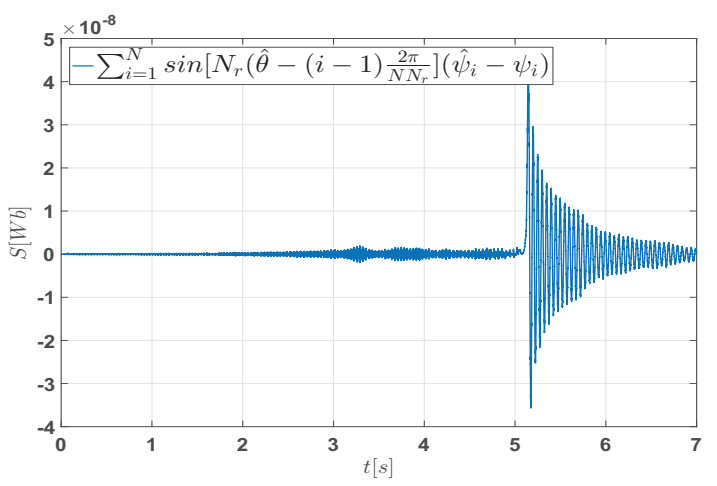

Figure 7: The sliding surface $(S)$.

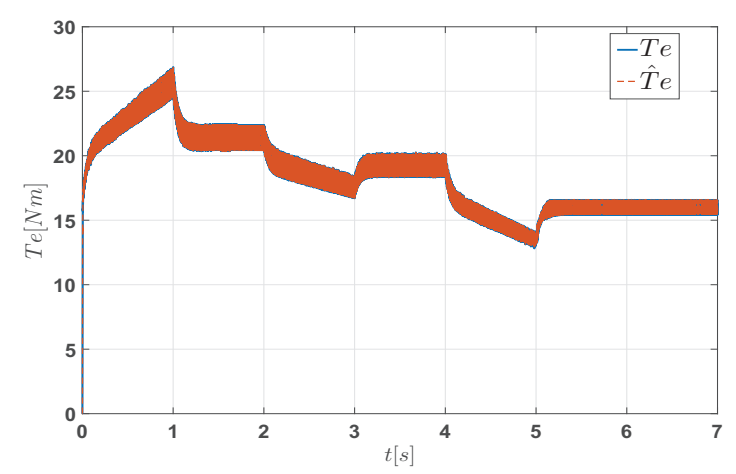

Figure 8: The electromagnetic torque estimation.

\section{Sliding Mode Observer with unknown load torque}

1) Observer design: The proposed SMO is extended to the case of an unknown load torque. In the reference [12], the estimation of speed acceleration is introduced to replace the dynamic model of the SRM. Using the SMO model developed by [12], the dynamic model of the SRM (2) is used to estimate the load torque. The same sliding surface defined in (16) is used, the SMO with unknown load torque model is defined as follows:

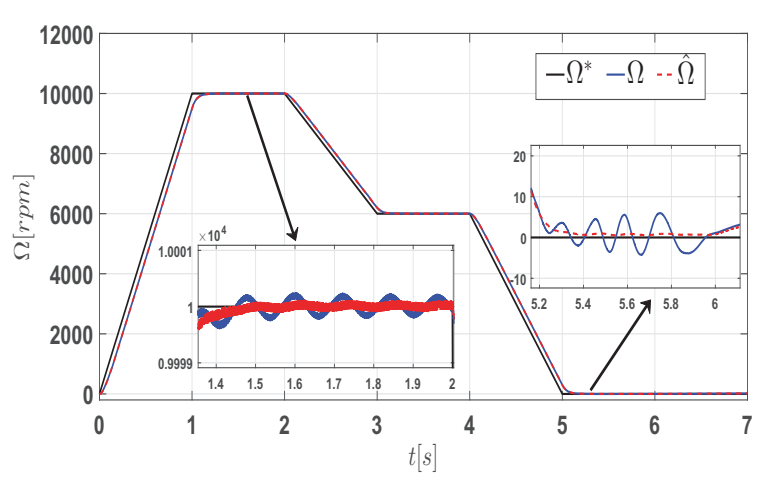

Figure 9: The rotor speed estimation with unknown $T_{L}$.

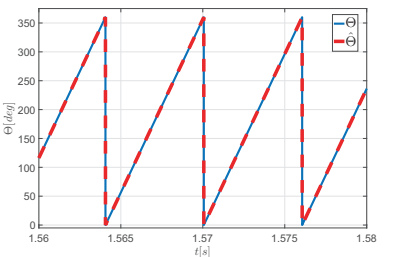

(a) At maximum speed.

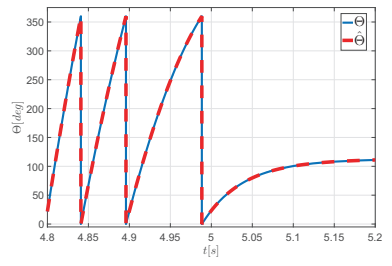

(b) At very low-speed.
Figure 10: The rotor position estimation with unkown $T_{L}$.

$$
\left\{\begin{array}{c}
\frac{\mathrm{d} \hat{\theta}}{\mathrm{dt}}=\hat{\Omega}+K_{\theta} \operatorname{sign}(S) \\
\frac{\mathrm{d} \hat{\Omega}}{\mathrm{dt}}=\hat{\alpha}+K_{\Omega} \operatorname{sign}(S) \\
\frac{\mathrm{d} \hat{\alpha}}{\mathrm{dt}}=K_{\alpha} \operatorname{sign}(S) \\
\hat{T}_{e}=T_{e}(\hat{\theta}, I) \\
\hat{T}_{L}=\hat{T}_{e}-f_{r} \hat{\Omega}-j \hat{\alpha}
\end{array}\right.
$$

Where, $\alpha$ and $\hat{\alpha}$ are respectively the angular acceleration and its estimated. $K_{\theta}, K_{\Omega}$ and $K_{\alpha}$ are SMO gains.

To find the observers gains, the same method as in [12] is applied.

2) Simulation results: The load torque is considered as an unknown input, The same speed profile used in section III-B2 is used. To minimize the chattering effect, the function $\operatorname{sing}(S)$ is replaced by $\operatorname{sat}(S)$ function in (20). The new SMO parameters are: $K_{\theta}=350, K_{\Omega}=550, K_{\alpha}=1500$ and $\phi=0.5$.

Fig.(9) displays the reference speed $\left(\Omega^{*}\right)$, the actual speed $(\Omega)$ and the estimated speed $(\hat{\Omega})$. On the whole speed range the performances are satisfactory. The designed SMO gives a very satisfying result of the position estimation over the entire speed range as shown in Fig.(10).

Fig.(11) shows the convergence of the sliding surface to zero in finite time even at low speed and standstill.

The electromagnetic torque of the machine and its estimated are shown in Fig.(12). One can see the good performances even during the transients. Moreover, the load torque estimation is also effective as it can be seen in Fig.(13). 


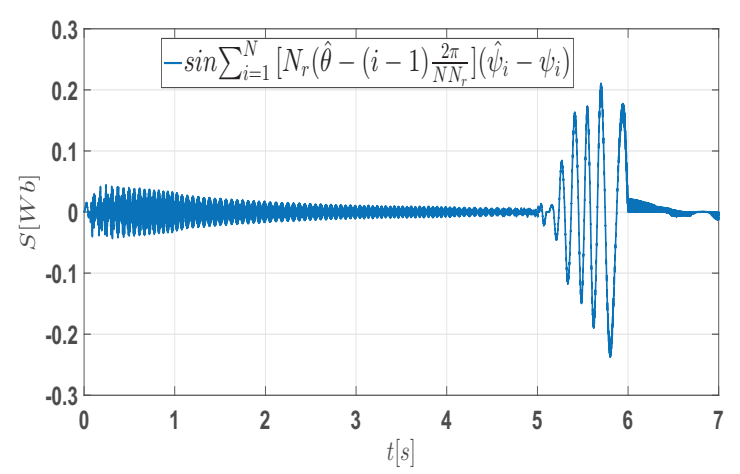

Figure 11: The sliding surface $(S)$ with unknown $T_{L}$.

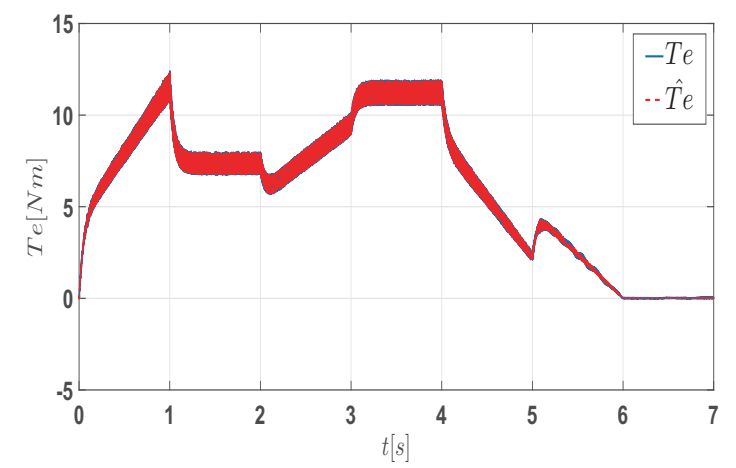

Figure 12: The torque estimation with unknown $T_{L}$.

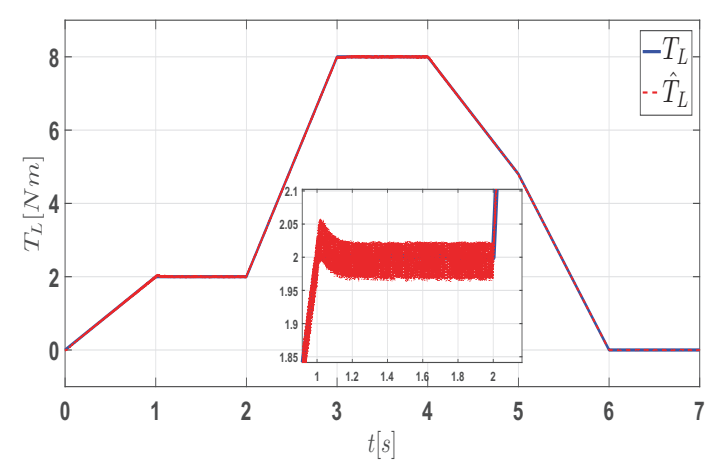

Figure 13: The load torque estimation $T_{L}$.

\section{CONCLUSION}

A SMO-based sensorless control algorithms of an Electric Vehicle propelled by a Switched Reluctance Machine with known and unknown load torque are developed and validated by simulation. Also, to ensure the stability of the proposed SMO at a low velocity of the machine and a standstill, a new dynamic sliding surface based on the machine flux estimation and the rotor position estimation is proposed. The simulation results confirm that the sliding mode observer estimates with a good precision estimation of rotor position, velocity and machine torque even at low velocity and at machine standstill. Indeed, the chattering of the estimated variables is comparatively insignificant on the performance of all the system where the SMO gains are designed with the boundary along the sliding manifold.

In future works, the equivalent inertia of the vehicle will be considered taking into account inertia of the vehicle load. Finally, the developed observer will be implemented and validated on a test bench of two electric machines. A SRM to emulate at a reduced scale the electric machine used in EV associated to the gearbox of a real vehicle and an asynchronous machine to emulate the vehicle load with a scale factor.

\section{APPENDIX}

\begin{tabular}{|l|l|}
\hline Parameter & Value \\
\hline Topology & $8 \mathrm{~S} / 6 \mathrm{R}$ \\
\hline Phase number & 4 \\
\hline Power supply (DC) & $250 \mathrm{~V}$ \\
\hline Maximum current & $61 \mathrm{~A}$ \\
\hline Nominal torque & $20 \mathrm{Nm}$ \\
\hline Nominal Power & $8 \mathrm{~kW}$ \\
\hline Maximum speed & $10000 \mathrm{rpm}$ \\
\hline Phase resistance $R$ & $0.0404 \mathrm{Ohm}$ \\
\hline Moment of inertia $J_{S R M}$ & $0.0043 \mathrm{Kg} / \mathrm{m} 2$ \\
\hline Friction $f_{r}$ & $0.005 \mathrm{Nm} / \mathrm{s}$ \\
\hline
\end{tabular}

\section{REFERENCES}

[1] Iqbal Husain, Electric and Hybrid Vehicles: Design fundamentals, CRC Press, 2003.

[2] J. D.Widmer, R. Martin, M. Kimiabeigi, "Electric vehicle traction motors without rare earth magnets," Sustainable Materials and Technologies, vol. 3, pp. 7-13, April 2015.

[3] K. Kiyota, T. Kakishima, A. Chiba and M. A. Rahman, "Cylindrical Rotor Design for Acoustic Noise and Windage Loss Reduction in Switched Reluctance Motor for HEV Applications," in IEEE Transactions on Industry Applications, vol. 52, no. 1, pp. 154-162, Jan.-Feb. 2016.

[4] N. Ouddah, M. Boukhnifer, A. Chaibet and E. Monmasson, "Robust controller designs of switched reluctance motor for electrical vehicle," 22nd Mediterranean Conference on Control and Automation, Palermo, 2014, pp. 212-217.

[5] R. Krishnan, Switched Reluctance Motor Drives: Modeling, Simulation, Analysis, Design, and Applications, 1 st ed., CRC Press, pp. 432, 2001

[6] B. Fahimi, G. Suresh and M. Ehsani, "Review of sensorless control methods in switched reluctance motor drives," Conference Record of the 2000 IEEE Industry Applications Conference. Thirty-Fifth IAS Annual Meeting and World Conference on Industrial Applications of Electrical Energy, Rome, vol.3, pp. 1850-1857, 2000.

[7] Lumsdaine, A. and Lang, J.H., State observers for variable-reluctance motors. IEEE Trans. Industrial Electronics, Vol. IE-37 (1990), No. 2, pp. 133-142.

[8] Y. Hassoun and M. B. Rifai, "Drive of senseless Switched Reluctance Motor (SRM) depending on Artificial Neural Networks," 2017 10th Jordanian International Electrical and Electronics Engineering Conference (JIEEEC), Amman, Jordan, 2017, pp. 1-9.

[9] C. Xia, F. Jia, M. Xue and H. Fang, "Commutation signal identification for switched reluctance motors based on fuzzy neural networks," 2008 IEEE International Conference on Automation and Logistics, Qingdao, 2008, pp. 700-704.

[10] R. A. McCann, M. S. Islam and I. Husain, "Application of a slidingmode observer for position and speed estimation in switched reluctance motor drives," in IEEE Transactions on Industry Applications, vol. 37, no. 1, pp. 51-58, Jan/Feb 2001.

[11] M. Lyapunov. The general problem of the stability of motion. Taylor and Francis, 1992.

[12] F. Peng, J. Ye, A. Emadi and Y. Huang, "Position Sensorless Control of Switched Reluctance Motor Drives Based on Numerical Method," in IEEE Transactions on Industry Applications, vol. 53, no. 3, pp. 21592168, May-June 2017. 